\title{
Programa de Triagem Neonatal para Hipotireoidismo Congênito no Nordeste do Brasil: Critérios Diagnósticos e Resultados
}

\begin{abstract}
RESUMO
Avaliamos as concentrações do TSH em papel-filtro colhido no calcanhar (TSHneo) de 48.039 crianças triadas do programa de triagem neonatal (PTN) para o hipotireoidismo congênito $(\mathrm{HC})$ de Sergipe, as concentrações de TSH, T4 total e T4 livre colhidas em sangue periférico nas crianças convocadas suspeitas de $\mathrm{HC}$, a idade nas diversas fases do programa, a cobertura e a freqüência do PTN de janeiro de 2005 a agosto de 2006, comparando-as com dados da literatura. Utilizamos para análise os seguintes parâmetros: média, mediana, coeficiente de variação e distribuição de freqüência. A idade da criança por ocasião da coleta em papel filtro no calcanhar foi $10 \pm 9$ dias (média \pm desvio-padrão) e a idade na realização do ensaio do TSHneo foi de $31 \pm 13$ dias. Em 2005, a cobertura do PTN, para o interior e para a capital de Sergipe, foi de $77 \%$ e $73 \%$, respectivamente. Verificamos que em $99,484 \%$ das crianças triadas as concentrações do TSH coletado em papel-filtro encontravam-se entre 0,01 e 5,20 $\mu \mathrm{U} / \mathrm{mL}$. As concentrações do TSH decrescem com o aumento da idade até estabilizar entre 11 e 15 dias de vida. Foram convocadas 248 crianças a partir do TSH coletado em papel-filtro (1/194). Na convocação, as concentrações do TSH, T4 e T4 livre coletado por punção venosa estavam normais em 119 crianças (1/404). A freqüência de $\mathrm{HC}$ suspeito foi de $1 / 485$ (99 casos), de $\mathrm{HC}$ foi de $1 / 6.005$ ( 8 casos) e de hipotiroxinemia foi de $1 / 16.013$ ( 3 casos). A terapia para o $\mathrm{HC}$ foi iniciada com $51 \pm 12$ dias. (Arq Bras Endocrinol Metab 2008;52/4:617-627)
\end{abstract}

Descritores: Hipotireoidismo congênito; Triagem neonatal; TSH; Tiroxina.

\begin{abstract}
Neonatal Screening Program for Congenital Hypothyroidism in Northeast of Brazil: Criteria, Diagnosis and Results.

It was evaluated the concentration of TSH in blood spot (TSHneo) of 48.039 children included in the Neonatal Screening Program (NSP) for Congenital Hypothyroidism $(\mathrm{CH})$ of Sergipe (SE), a state in the northeast of Brazil. It was also evaluated the concentration of serum TSH, total T4 and free T4 in the recalled children suspicious of having $\mathrm{CH}$, their age in several phases of the program, the covering and frequency of the NSP in the cases from January 2005 to August 2006, comparing them with literature data. The following parameters were used or the analysis: mean, standard deviation, median, coefficient of variation and frequency distribution. The children's age at the collection in filter-paper specimen was $10 \pm 9$ days (Mean \pm SD) and the TSHneo execution assay was done in the period of $31 \pm 13$ days. In 2005 the covering by the NSP was about $77 \%$ in the countryside and $73 \%$ in Aracaju, the capital of Sergipe. It was verified that in $99,484 \%$ of the children included in the screening, the TSHneo varied from 0,01 to $5,20 \mu \mathrm{U} / \mathrm{ml}$, decreasing according to the age and stabilizing when they were between 11 and 15 days. 248 children were recalled from the TSHneo (1/194). The concentrations of TSH, T4 and free T4 collected by venous puncture were normal in 119 children (1/404). The frequency of suspected $\mathrm{CH}$ was $1 / 485$ (99 cases), of $\mathrm{CH}$ was $1 / 6005$ ( 8 cases) and of hypothyroxinemia was $1 / 16013$ ( 3 cases). Therapy for $\mathrm{CH}$ began within $51 \pm 12$ days. (Arq Bras Endocrinol Metab 2008;52/4:617-627)
\end{abstract}

Keywords: Congenital hypothyroidism; Neonatal screening; TSH; Thyroxin. artigo original

\author{
ANTÔNIO R. O. RAMALHO \\ ROBERTO J. R. RAMALHO \\ CARLA R. P. OLIVEIRA \\ ELENILDE G. SANTOS \\ MARIO C. P. OliveIRA \\ Manuel H. Aguiar-Oliveira
}

Divisão de Endocrinologia do Departamento de Medicina da Universidade Federal de Sergipe (UFS), Aracaju, SE, Brasil.

Recebido em 14/08/2007 Aceito em 4/4/2008 


\section{INTRODUÇÃo}

$\mathrm{O}$ EXAME DE CASOS CLÍNICOS e a tentativa de possível explicação para o caso clínico em questão é prática antiga na experiência médica. A clínica foi elemento de acumulação positiva, e o constante olhar sobre o doente, milenar, e, no entanto, novo, permitiu à medicina evoluir. Os instrumentos que se aplicam ao corpo a que se dirige a observação foram incrementados. A profundidade que o olhar do médico percorre foi ampliada antecipando a atenção à saúde com medidas preventivas (1).

A história da medicina está cheia de referências ocasionais à tireóide, mas o estudo científico da glândula só teve início em época recente quando Thomas Curling em 1850 fez a observação clínica da ausência de tireóide em cretinos. Foi a primeira observação da relação entre o quadro clínico e o anatômico relacionado à glândula endócrina (2).

A evolução na metodologia das dosagens hormonais, combinadas com a observação clínica, permitiu que medidas preventivas de saúde pública fossem implementadas. Em 1974, em Quebec e Pittsburg foi proposto o programa de triagem neonatal (PTN) para o hipotireoidismo congênito (HC) como forma de diagnóstico pré-clínico que propiciaria o tratamento precoce prevenindo o retardo mental dos casos identificados e tratados $(3,4)$. Estudos de Fisher e Klein, em 1981 (5), demonstraram as concentrações de hormônios tireoidianos e do TSH no período pré-natal e pós-natal com redução progressiva do TSH à medida que há o aumento da idade da criança até a estabilização. Naruse e col., em 1986 (6), estudaram os níveis do TSH no período pós-natal pelo método de radioimunoensaio e enzimaimunoensaio em programa de triagem neonatal para o HC no Japão.

A introdução dos programas de triagem neonatal representa importante instrumento de prevenção de retardo mental com adequada e tempestiva terapia substitutiva com L-tiroxina para os casos de HC, propiciando o desenvolvimento neuropsíquico da criança e de critérios para sua classificação (4,7-21).

No Brasil, em 2001, foi criado o Programa Nacional de Triagem Neonatal pelo Ministério da Saúde. A nova política da triagem neonatal foi propiciada no Brasil por meio da criação de norma pelo Ministério da Saúde (Portaria n ${ }^{\circ} 822 / 2001$ ), que disciplinou os programas de triagem, redundando na nomeação do
Hospital Universitário da Universidade Federal de Sergipe (HU/UFS) como único serviço público de referência em triagem neonatal no Estado de Sergipe, possibilitando estudos populacionais.

O primeiro objetivo do presente trabalho foi avaliar as concentrações de TSH colhido em papel-filtro do calcanhar em uma população de 48.039 crianças triadas pelo PTN no Estado de Sergipe, enfatizando a utilização dos pontos de corte para o estudo dos casos com concentrações limítrofes nas diversas faixas etárias. O outro objetivo foi a avaliação das concentrações do TSH, T4 e T4 livre séricas colhidas por punção venosa das crianças convocadas para confirmação e classificação do diagnóstico do HC.

\section{CASUÍSTICA E MÉTODOS}

Foi realizado um estudo transversal nas crianças triadas mensalmente pelo PTN-SE. Foram obtidos os quantitativos (48.039 crianças) das triagens realizadas no Serviço Público (HU/UFS), referentes ao período de janeiro de 2005 a agosto de 2006.

Utilizamos os seguintes procedimentos: quantificou-se o número de crianças triadas na capital e no interior e calculou-se a média aritmética, mediana, o percentual e o desvio-padrão das idades das crianças triadas e das concentrações do TSH coletados em papel-filtro (tipo SS 903). O sangue foi colhido com lanceta do calcanhar das crianças, após o nascimento, e o TSH neonatal (TSHneo) foi dosado pelo método imunofluorimétrico da PerkinElmer Life and Analytical Sciences, Wallac, Turku, Finland. Foram analisadas as variações máxima e mínima das concentrações do TSHneo e das idades das crianças triadas nas fases do PTN-SE; o coeficiente de variação (cv - desvio-padrão vezes 100 dividido pela média) da concentração do TSHneo e das idades das crianças; e a distribuição de freqüência percentual. Fizemos a representação gráfica da curva de distribuição de freqüência da concentração do TSHneo por meio de análises de registros por intervalos de concentrações e sua variação máxima e mínima nos quadrimestres analisados $(6,22)$.

Foram calculadas a incidência e a cobertura de HC segundo critérios estabelecidos, dividindo o número de casos de HC selecionados pela quantidade de crianças triadas e a quantidade de crianças triadas pela quantidade de nascidos vivos respectivamente (NV/ SINASC/2004 igual a 35.827). 
Os pontos de corte para o TSHneo para definir se a criança era suspeita de $\mathrm{HC}$ e prosseguir a investigação clínica e laboratorial realizada pelo PTN-SE foram calculados a partir dos limites superiores do intervalo de confiança de 95\%, segundo metodologia descrita (22-24). A média e o desvio-padrão dos resultados foram calculados para todas as crianças triadas e para o grupo com a idade na coleta de 2 a 6 dias. As crianças com TSHneo maior que $12,9 \mu \mathrm{U} / \mathrm{mL}$, concentração recomendada no manual de instruções revisado em novembro de 2002 do kit B032-312 do TSH da Wallac/ PerkinElmer Life Sciences, Wallac Ou, Turku, Finland (24), não foram incluídas para o cálculo da média, desvio-padrão, e, conseqüentemente, do ponto de corte, mas estão indicadas na tabela 1 .

Os valores do TSHneo, nos casos alterados (acima do ponto de corte), foram repetidos utilizando-se a média aritmética das duplicatas da mesma amostra. As amostras colhidas em papel-filtro são acondicionadas em refrigerador a $4^{\circ}$ centrígados no posto de saúde local em todos os municípios sergipanos. O transporte do posto de coleta ao laboratório para o Estado de Sergipe não ultrapassa seis horas e é realizado com as amostras na temperatura ambiente.

Para finalidade deste trabalho, o PTN-SE considerou criança com resultado alterado para o HC quando a concentração do TSHneo colhida em papel-filtro está superior a $5,2 \mu \mathrm{U} / \mathrm{mL}$, que corresponde aproximadamente à média das concentrações mais quatro desvios-padrão das concentrações, o que equivale a $0,52 \%$ das crianças triadas. Esse valor segue tendência de redução dos pontos de corte inicialmente sugeridos (4,6,23-27). Foi considerada a faixa de segurança mais ampla para evitar ao máximo os falso-negativos (4,28-32). A criança é encaminhada ao ambulatório multiprofissional do HU/UFS para seguimento, devendo ser confirmado o HC. Após a convocação da criança com resultado alterado para o HC, são colhidos exames séricos por punção venosa para a medida do T4 total, T4 livre e TSH pelo método imunofluorimétrico.

O PTN-SE considerou as seguintes possibilidades a partir do teste de triagem neonatal alterado ( $\mathrm{TSH}$ neo colhido no calcanhar em papel-filtro maior do que o ponto de corte):

a) criança descartada de suspeita de HC na convocação quando TSH, T4 total e livre séricos coletado por punção venosa estão normais; b) hipotireoidismo congênito suspeito na convocação quando o TSH sérico coletado por punção venosa está elevado e o T4 total e/ou T4 livre séricos coletado por punção venosa estão normais;

c) hipotireoidismo congênito quando o TSH sérico coletado por punção venosa está elevado e o T4 total e T4 livre séricos coletado por punção venosa estão baixos;

d) hipotiroxinemia quando o TSH sérico coletado por punção venosa está normal e o T4 total e/ou livre séricos coletado por punção venosa estão baixos;

e) casos pendentes: pacientes que não compareceram na convocação.

Os valores normais de TSH, T4 total e T4 livre séricos obtidos na convocação por punção venosa para finalidade deste trabalho são menor que $4,2 \mu \mathrm{U} / \mathrm{mL}$ e maiores que $7,20 \mu \mathrm{g} / \mathrm{dL}$ e $0,79 \mathrm{ng} / \mathrm{dL}$, respectivamente. Para a apuração dos dados utilizou-se a planilha eletrônica Excel ${ }^{\circledR}$ (Microsoft). Para comparação entre os grupos usou-se o teste $t$ de Student com $\mathrm{p}$ menor que 0,05 .

\section{RESULTADOS}

Os dados de 48.039 crianças triadas $(23,71 \%$ capital - 11.392 crianças e $76,29 \%$ interior -36.647 crianças) de janeiro de 2005 a agosto de 2006 com TSHneo agrupados nos quadrimestres analisados segundo o intervalo de concentração de TSH estão mostrados na tabela 1 e na figura 1. Em 2005 a cobertura do PTNSE foi de $76,28 \%(73,10 \%$ na capital e $77,36 \%$ no interior de Sergipe). Verificamos que em 99,484\% das crianças triadas as concentrações do TSHneo coletado em papel-filtro se encontram entre o intervalo de 0,01 até $5,20 \mu \mathrm{U} / \mathrm{mL}$ (tabela $\mathrm{l}$ ). Verificamos, após a convocação, os resultados dos exames confirmatórios séricos obtidos por punção venosa das crianças com resultados do TSHneo coletado do calcanhar em papel-filtro acima do ponto de corte. A tabela 1 mostra as possibilidades de inclusão das crianças com o TSHneo alterado e os exames séricos confirmatórios. Foram descartadas 119 crianças $(47,98 \%$ das convocadas). Verificamos incidência de HC suspeitos de $1 / 485$, HC de 1/6.005 e hipotiroxinemia de 1/16.013. Houve percentual de pendências de $7,66 \%$ dos casos alterados. 


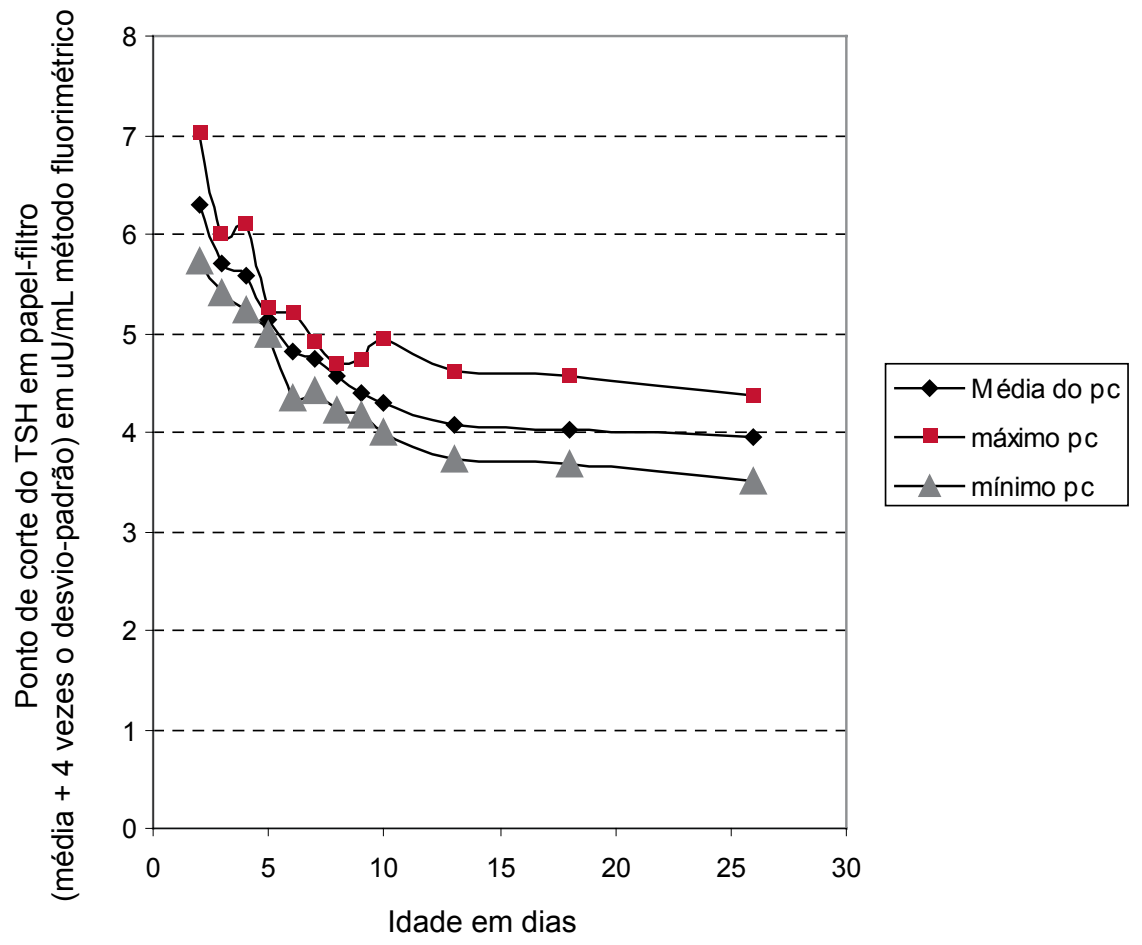

Figura 1. Percentual de crianças triadas no PTN-SE de janeiro de 2005 a agosto de 2006 em intervalos de concentrações de 48.026 crianças com medida do TSH coletado do calcanhar em papel-filtro, exceto aquelas com concentrações acima de $12,9 \mu \mathrm{U} / \mathrm{mL}$, analisados por quadrimestres e sua variação máxima e mínima.

A idade de todas as crianças na coleta de sangue na convocação para confirmação do diagnóstico foi de 49,63 $\pm 19,32$ dias, mediana de 45 dias, máximo de 128 dias e mínimo de 10 dias. Até 60 dias de idade $77,06 \%$ das crianças foram convocadas.

Foram detectados 99 casos de HC suspeitos, com idade na coleta do TSHneo de 7,37 $\pm 5,87$ dias (máximo de 31 e mínimo de 2 dias) e concentração do TSHneo de 8,03 4,77 $\mu \mathrm{U} / \mathrm{mL}$ (máximo de 30,80 e mínimo de $5,21 \mu \mathrm{U} / \mathrm{mL}$ ). O TSH na convocação foi coletado por punção venosa com 48,90 \pm 19,72 dias (máximo de 128 e mínimo de 10 dias, 88 casos com mais de 30 dias) e concentração do TSH de 7,48 \pm $8,24 \mu \mathrm{U} / \mathrm{mL}$ (máximo de 83,40 e mínimo de 4,24 $\mu \mathrm{U} / \mathrm{mL})$. Dos 99 casos de HC suspeito, 12 (12,12\%) casos tiveram TSHneo maior que $10 \mu \mathrm{U} / \mathrm{mL}$ e em 3 $(3,03 \%)$ deles o TSH sérico na convocação obtido por punção venosa foi maior que $10 \mu \mathrm{U} / \mathrm{mL}$. Dos $87 \mathrm{ca}$ sos com TSHneo menor que $10 \mu \mathrm{U} / \mathrm{mL}, 8(9,19 \%)$ tiveram TSH sérico na convocação obtido por punção venosa maior que $10 \mu \mathrm{U} / \mathrm{mL}$. Dos 99 casos de $\mathrm{HC}$ suspeito, $11(11,11 \%)$ casos tiveram TSH sérico na convocação obtido por punção venosa maior que 10 $\mu \mathrm{U} / \mathrm{mL}$, variando de 10,20 a $83,40 \mu \mathrm{U} / \mathrm{mL}$ com idade na coleta sérica variando de 33 a 85 dias.

Foram detectados 8 casos de HC com idade na coleta do TSHneo de 14,25 $\pm 11,02$ dias (máximo de 36 e mínimo de 4 dias) e concentração do TSHneo de $110,39 \pm 137,32 \mu \mathrm{U} / \mathrm{mL}$ (máximo de 384,00 e mínimo de $9,13 \mu \mathrm{U} / \mathrm{mL})$. O TSH sérico na convocação foi coletado por punção venosa com 55,75 $\pm 17,19$ dias (máximo de 85 e mínimo de 36 dias) e concentração do TSH de 233,86 $\pm 123,01 \mu \mathrm{U} / \mathrm{mL}$ (máximo de 364,00 e mínimo de $66,20 \mu \mathrm{U} / \mathrm{mL}$ ). Dos 8 casos, 1 $(12,5 \%)$ teve TSHneo menor que $10 \mu \mathrm{U} / \mathrm{mL}$, e 3 $(37,5 \%)$ casos entre 10 e $20 \mu \mathrm{U} / \mathrm{mL}$.

Foram detectados três casos de hipotiroxinemia com idade na coleta do TSHneo de 3,00 dias $\pm 1,00$ dia (máximo de 4 e mínimo de 2 dias) e concentração 
Tabela 1. Distribuição de freqüência por intervalo de concentrações do TSHneo em crianças do PTN-SE de janeiro de 2005 a agosto de 2006 e os casos selecionados com TSHneo acima do ponto de corte e suas possibilidades diagnósticas após as dosagens venosas.

\begin{tabular}{|c|c|c|c|c|c|c|c|c|}
\hline \multirow{2}{*}{$\begin{array}{c}\text { TSHneo } \mu \mathrm{U} / \\
\mathrm{mL}\end{array}$} & \multirow{2}{*}{$\begin{array}{l}\text { Qtd. } \\
\text { total }\end{array}$} & \multirow[t]{2}{*}{$\% A$} & \multirow{2}{*}{$\begin{array}{c}\text { Total } \\
\text { selecionado }\end{array}$} & \multicolumn{5}{|c|}{ Possibilidades } \\
\hline & & & & Descartado & HC suspeito & $\mathrm{HC}$ & Hipotiroxinemia & Pendência \\
\hline 0,01 a 1,00 & 26.049 & 54,225 & 0 & 0 & 0 & 0 & 0 & 0 \\
\hline 1,01 a 2,00 & 15.099 & 85,655 & 0 & 0 & 0 & 0 & 0 & 0 \\
\hline 2,01 a 3,00 & 4.539 & 95,100 & 0 & 0 & 0 & 0 & 0 & 0 \\
\hline 3,01 a 4,00 & 1.468 & 98,156 & 0 & 0 & 0 & 0 & 0 & 0 \\
\hline 4,01 a 5,20 & 638 & 99,484 & 0 & 0 & 0 & 0 & 0 & 0 \\
\hline 5,21 a 6,0 & 81 & 99,653 & 81 & 42 & 30 & 0 & 1 & 8 \\
\hline 6,01 a 7,00 & 71 & 99,800 & 71 & 34 & 28 & 0 & 1 & 8 \\
\hline 7,01 a 8,00 & 32 & 99,867 & 32 & 17 & 13 & 0 & 1 & 1 \\
\hline 8,01 a 9,00 & 25 & 99,919 & 25 & 15 & 8 & 0 & 0 & 2 \\
\hline 9,01 a 10,00 & 14 & 99,948 & 14 & 5 & 8 & 1 & 0 & 0 \\
\hline 10,01 a 11,00 & 3 & 99,954 & 3 & 0 & 2 & 1 & 0 & 0 \\
\hline 11,01 a 12,00 & 5 & 99,965 & 5 & 2 & 3 & 0 & 0 & 0 \\
\hline 12,01 a 12,99 & 3 & 99,971 & 3 & 3 & 0 & 0 & 0 & 0 \\
\hline 13,00 & 1 & 99,973 & 1 & 0 & 1 & 0 & 0 & 0 \\
\hline 13,30 & 1 & 99,975 & 1 & 0 & 1 & 0 & 0 & 0 \\
\hline 13,36 & 1 & 99,977 & 1 & 0 & 0 & 1 & 0 & 0 \\
\hline 13,80 & 1 & 99,979 & 1 & 0 & 1 & 0 & 0 & 0 \\
\hline 19,70 & 1 & 99,982 & 1 & 0 & 0 & 1 & 0 & 0 \\
\hline 27,50 & 1 & 99,984 & 1 & 0 & 1 & 0 & 0 & 0 \\
\hline 28,40 & 1 & 99,986 & 1 & 0 & 1 & 0 & 0 & 0 \\
\hline 30,70 & 1 & 99,988 & 1 & 0 & 1 & 0 & 0 & 0 \\
\hline 30,80 & 1 & 99,990 & 1 & 0 & 1 & 0 & 0 & 0 \\
\hline 53,20 & 1 & 99,992 & 1 & 0 & 0 & 1 & 0 & 0 \\
\hline 116,10 & 1 & 99,994 & 1 & 1 & 0 & 0 & 0 & 0 \\
\hline 172,00 & 1 & 99,996 & 1 & 0 & 0 & 1 & 0 & 0 \\
\hline 221,00 & 1 & 99,998 & 1 & 0 & 0 & 1 & 0 & 0 \\
\hline 384,00 & 1 & 100,000 & 1 & 0 & 0 & 1 & 0 & 0 \\
\hline Total & 48.039 & & 248 & 119 & 99 & 8 & 3 & 19 \\
\hline $\begin{array}{c}\% \text { do } \\
\text { selecionado }\end{array}$ & & & 100 & 49,98 & 39,92 & 3,23 & 1,21 & 7,66 \\
\hline Incidência & & & $1 / 194$ & $1 / 404$ & $1 / 485$ & $1 / 6.005$ & $1 / 16.013$ & $1 / 2.528$ \\
\hline \% do triado & & & 0,516 & 0,248 & 0,206 & 0,017 & 0,006 & 0,039 \\
\hline
\end{tabular}


do TSHneo de 6,60 $\pm 1,17 \mu \mathrm{U} / \mathrm{mL}$ (máximo de 7,52 e mínimo de $5,28 \mu \mathrm{U} / \mathrm{mL}$ ). O TSH sérico na convocação foi coletado por punção venosa com 57,33 \pm 12,50 dias (máximo de 70 e mínimo de 45 dias), concentração do TSH de $2,92 \pm 0,53 \mu \mathrm{U} / \mathrm{mL}$ (máximo de 3,51 e mínimo de $2,48 \mu \mathrm{U} / \mathrm{mL}$ ).

A tabela 2 mostra as diversas idades da criança na triagem neonatal coletada em papel-filtro nos respectivos quadrimestres. A média da idade da criança na coleta da amostra do TSHneo variou de 9,9 dias a 11,0 dias, e o desvio-padrão de 7,7 dias a 12,6 dias. A média da concentração do TSHneo nos períodos analisados variou de $1,10 \mu \mathrm{U} / \mathrm{mL}$ a $1,24 \mu \mathrm{U} / \mathrm{mL}$ e o desvio-padrão de 0,89 a $1,10 \mu \mathrm{U} / \mathrm{mL}$. Verificamos que as médias dos TSHneos na faixa de idade de 2 a 6 dias são maiores.

A idade da criança na coleta do TSHneo variou entre 2 e 312 dias. O número e a idade das crianças por ocasião da coleta de sangue do calcanhar em papelfiltro no período observado para medida do TSHneo foi de: 10.970 crianças de 2 a 5 dias de vida $(22,84 \%)$; 18.473 crianças de 6 a 10 dias de vida $(38,45 \%) ; 8.152$ crianças de 11 a 15 dias de vida $(16,97 \%) ; 2.739$ crianças de 16 a 20 dias de vida $(5,70 \%) ; 2.784$ crianças de 21 a 30 dias de vida $(5,80 \%) ; 753$ crianças de 31 a 40 dias de vida $(1,57 \%) ; 237$ crianças de 41 a 50 dias de vida $(0,48 \%) ; 95$ crianças de 51 a 60 dias de vida $(0,20 \%)$; maior que 60 dias $(7,99 \%)$. De 2 a 30 dias e de 2 a 60 dias de idade na coleta do TSHneo foram triadas $89,76 \%$ e $92,01 \%$ das crianças, respectivamente. O tempo da coleta à realização do ensaio das amostras de TSHneo nos quadrimestres estudados variou entre 16,6 dias a 23,7 dias.

Analisando os resultados de 43.118 crianças triadas, no intervalo de idade de 2 a 30 dias, verificamos que a concentração do TSHneo decresce com a idade até se estabilizar entre 11 e 15 dias de vida. Foram também representadas variação máxima e variação mínima do ponto de corte nos quadrimestres analisados (figura 2).

Tabela 2. Idade da coleta em dias e concentrações de TSH neonatal com idade de 2 a 6 dias e em todas as idades entre janeiro de 2005 a agosto de 2006.

\begin{tabular}{|c|c|c|c|c|c|c|c|}
\hline Quadrimestre/ano & $\begin{array}{l}\text { Quantidade total } \\
\text { de coletas }\end{array}$ & $\begin{array}{l}\text { Quantidade } \\
\text { de coletas de } \\
2 \text { a } 6 \text { dias }\end{array}$ & Variável & $\begin{array}{l}\text { IDC } \\
\text { total }\end{array}$ & $\begin{array}{c}\text { IDC } \\
\text { coleta } 2 \\
\text { a } 6 \text { dias }\end{array}$ & $\begin{array}{l}\text { TSHneo } \mu \mathrm{U} / \\
\mathrm{mL} \text { total }\end{array}$ & $\begin{array}{l}\text { TSHneo } \mu \mathrm{U} / \mathrm{mL} \\
\text { coleta } 2 \text { a } 6 \\
\text { dias }\end{array}$ \\
\hline \multirow[t]{2}{*}{$1^{\circ}$ quadrimestre/2005 } & \multirow[b]{2}{*}{7.646} & \multirow[t]{2}{*}{2.334} & Média & 11,0 & 4,5 & 1,24 & 1,38 \\
\hline & & & DP & 12,6 & 1,1 & 1,10 & 1,10 \\
\hline \multirow[t]{2}{*}{$2^{\circ}$ quadrimestre/2005 } & \multirow[b]{2}{*}{10.723} & \multirow[t]{2}{*}{3.377} & Média & 10,8 & 4,7 & 1,13 & 1,32 \\
\hline & & & DP & 8,6 & 1,1 & 0,89 & 0,98 \\
\hline \multirow[t]{2}{*}{$3^{\circ}$ quadrimestre/2005 } & \multirow[b]{2}{*}{8.963} & \multirow[t]{2}{*}{3.164} & Média & 10,0 & 4,7 & 1,21 & 1,36 \\
\hline & & & DP & 10,0 & 1,0 & 0,99 & 1,11 \\
\hline \multirow[t]{2}{*}{$1^{\circ}$ quadrimestre/2006 } & \multirow[b]{2}{*}{10.137} & \multirow[t]{2}{*}{3.372} & Média & 9,9 & 4,7 & 1,10 & 1,28 \\
\hline & & & DP & 7,7 & 1,0 & 0,96 & 1,13 \\
\hline \multirow[t]{2}{*}{$2^{\circ}$ quadrimestre/2006 } & \multirow[b]{2}{*}{10.570} & \multirow[t]{2}{*}{3.039} & Média & 10,2 & 4,7 & 1,11 & 1,30 \\
\hline & & & DP & 8,2 & 1,1 & 0,95 & 1,08 \\
\hline \multirow[t]{3}{*}{ Período total } & \multirow{3}{*}{48.039} & \multirow[t]{3}{*}{$15.304^{*}$} & Média & 10,4 & 4,7 & 1,16 & 1,33 \\
\hline & & & DP & 9,4 & 1,1 & 0,98 & 1,08 \\
\hline & & & $\begin{array}{l}\text { Coeficiente } \\
\text { de } \\
\text { variação }\end{array}$ & 90,6 & 23,4 & 84,6 & 81,2 \\
\hline
\end{tabular}

* $31,86 \%$ do total de 48.039 crianças triadas; DP: desvio-padrão; IDC: idade da criança na coleta em dias para medida do TSHneo; TSHneo: TSH neonatal medido pelo método imunofluorimétrico colhido do calcanhar em papel-filtro. 


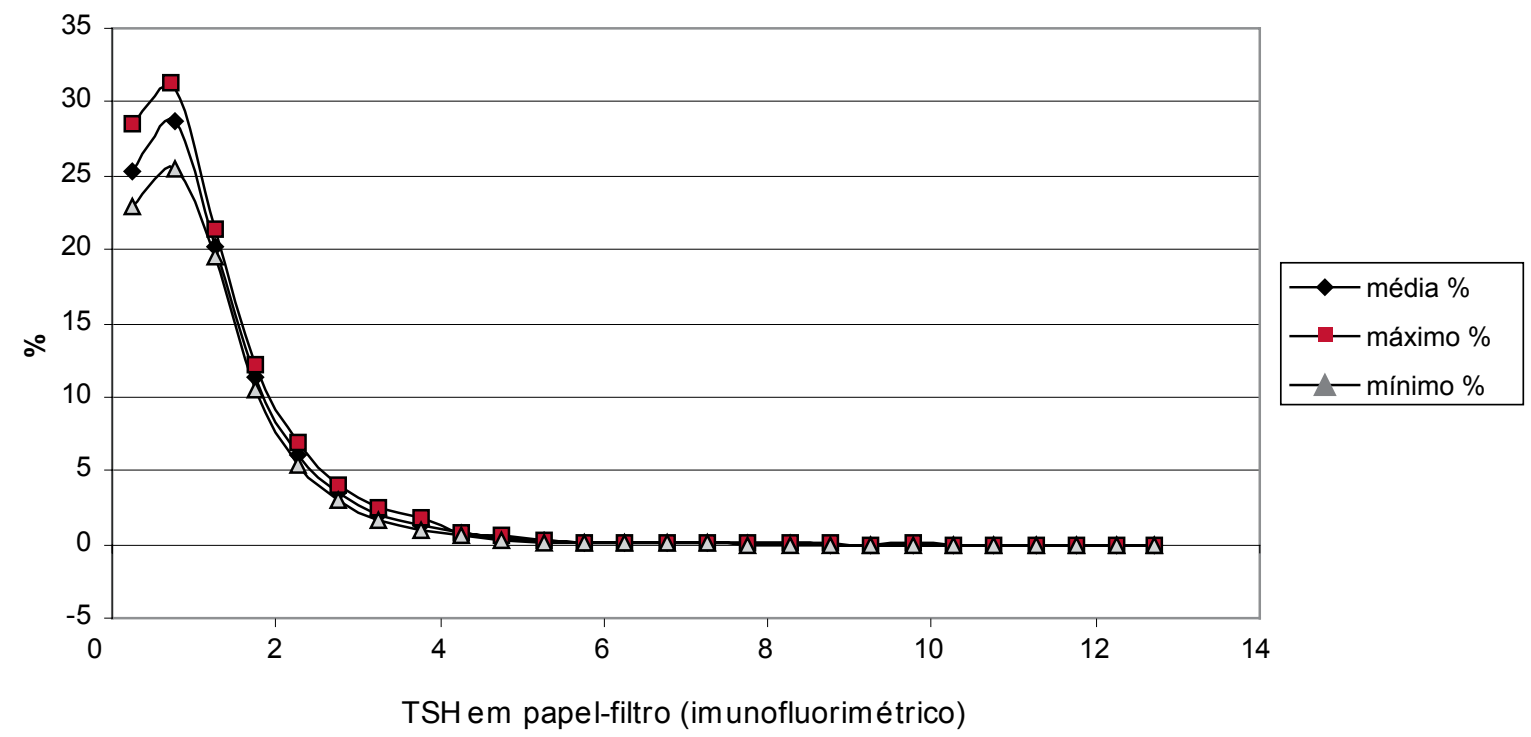

Figura 2. Variação do ponto de corte (pc) de $43.118 \mathrm{TSH}$ em papel-filtro de crianças triadas no PTN-SE por quadrimestre de janeiro de 2005 a agosto de 2006 e por idade na coleta de 2 a 30 dias.

\section{DISCUSSÃo}

O HC quando não tratado tempestiva e adequadamente contribui para retardo mental, disfunções do crescimento e do desenvolvimento neuropsicomotor da criança $(3,21)$.

O desenvolvimento de políticas e programas para melhorar a qualidade do crescimento e desenvolvimento infantil das crianças portadoras de HC poderá ser aperfeiçoado com base em evidências nas informações dos considerados suspeitos e portadores de HC. O risco estatístico de seqüelas é bem definido naquelas crianças com HC não tratado com TSHneo maior que $20 \mu \mathrm{U} / \mathrm{mL}$ medido pelo método imunofluorimétrico (4,26-31).

Contudo, diversos métodos de triagem são atualmente utilizados para selecionar casos suspeitos de HC. O importante é escolher o que melhor se adapta às condições de saúde pública local (4,26-37).

Existem diversas questões a serem consideradas na definição dos pontos de corte do TSHneo que traduzem nível seguro para definir a criança sadia ou suspeita de HC e sujeita a alterações do crescimento e desenvolvimento. A primeira é a idade da criança, quando é realizada a coleta do exame de triagem. A segunda é o tempo para a realização do ensaio. Os padrões de TSHneo estabelecidos em muitos progra- mas são construídos com base na variação da idade da coleta do recém-nascido, de 2 a 7 dias com poucos dias para realização do ensaio. Na prática, os programas de triagem no Brasil executam a coleta do exame e o ensaio com prazos maiores, criando um problema metodológico para definir o padrão para essa nova realidade $(4,5,36-46)$. Sabe-se que há redução da média das concentrações do TSH nos primeiros dias de vida até sua estabilização (5), e amostras estocadas sofrem inativação com o tempo. Nossos dados mostram a redução das concentrações do TSHneo com o aumento da idade da criança na coleta, o que pode contribuir para pontos de corte menores quando o teste de triagem é colhido mais tarde $(5,39)$. Existe a variação sazonal e circadiana que altera a concentração do TSH, mas no Nordeste do Brasil a temperatura é alta, a variação da temperatura ambiente é pequena e os exames em Sergipe são coletados pelo dia $(5,27,47,48)$. A terceira questão é a diversidade étnica que é confirmada pelas diferenças de incidência entre os povos $(48,49)$. A quarta é a diversidade ambiental e cultural influindo na concentração de iodo ingerido na alimentação $(14,33,49,50)$. Existem diferenças nas concentrações do TSH e do T4 neonatal relativas às taxas de iodo do solo que influenciam nas taxas de iodo alimentar como também os programas de adição do iodo ao sal de cozinha (51-56). 
O padrão utilizado para diferenciar a criança sadia da suspeita de HC deve-se guiar pela possibilidade mínima de erro de diagnóstico do programa de triagem neonatal, que poderá levar à posterior seqüela indelével na criança não identificada com $\mathrm{HC}(57,58)$.

O PTN-SE considera resultados alterados em crianças com TSHneo acima do ponto de corte estabelecido $(4,6,24-29,37,57)$. Na população-alvo, a freqüência de crianças descartadas após convocação foi de $1 / 404$. Como a convocação para confirmação do diagnóstico foi tardia (em geral 49,63 $\pm 19,32$ dias, mediana de 45 dias), é possível que entre essas crianças haja portadores de hipertirotropinemia transitória $(19,52)$. Com o ponto de corte de $5,2 \mu \mathrm{U} / \mathrm{mL}$ foi possível identificar casos que não poderiam ser identificados com o ponto de corte maior. Se fosse utilizada a concentração de $20 \mu \mathrm{U} / \mathrm{mL}$, como ponto de corte, para iniciar investigação de HC, deixaríamos de considerar 95 casos de suspeitos de HC, 4 casos de HC e 3 casos de hipotiroxinemia. Muitos programas de triagem neonatal reduziram seus pontos de corte para iniciar investigação mais ampla (4,25-27,36,59-64). Segundo consenso da American Academy of Pediatrics/2006, quando o T4 sérico é normal e há elevação do TSH sérico que persiste mais alto do que $10 \mathrm{mU} / \mathrm{L}$ depois das duas primeiras semanas de idade, o resultado é anormal. Nesse caso, a criança deve ser tratada. Se não tratada, deve ser repetida a dosagem do TSH e T4 livre em 2 a 4 semanas. Crianças com elevações do TSH entre 6 e $10 \mathrm{mU} / \mathrm{L}$, que persistem após o primeiro mês de vida, é assunto controverso. Caso a decisão seja por tratá-las, a retirada da L-tiroxina deveria ser realizada aos 3 anos de idade para reavaliação, em virtude do risco de se tratar de crianças eutireóideas (4). Em Sergipe, as crianças convocadas chegam ao $\mathrm{HU} / \mathrm{UFS}$ para confirmação do diagnóstico e início do tratamento com idade de 49,63 \pm 19,32 dias, mediana de 45 dias, máximo de 128 dias e mínimo de 10 dias. Se considerarmos como HC os 11 casos com TSH sérico na convocação maior que 10 $\mu \mathrm{U} / \mathrm{mL}$ com T4 e T4 livre normais, a incidência passaria a $1 / 2.528$ que é compatível com alguns relatos da literatura $(65,66)$.

Calaciura e col. (2002) (29) analisaram 56 crianças falso-positivas para o HC (convocação com T4 livre normal e TSH discretamente elevado) no screening neonatal (grupo I com TSH sérico na convocação < 5 $\mu \mathrm{U} / \mathrm{mL}$; grupo II com TSH sérico na convocação entre 5 e $11,7 \mu \mathrm{U} / \mathrm{mL}$ ). Com 16 a 44 meses de idade, as concentrações séricas do TSH foram significativa- mente mais altas nas 56 crianças falso-positivas comparadas a 65 crianças com TSNneo normal $(4,4 \pm 2,2$ $\mu \mathrm{U} / \mathrm{mL}$ e $1,4 \pm 0,8 \mu \mathrm{U} / \mathrm{mL})$. A freqüência do $\mathrm{HC}$ subclínico foi de $36 \%$ nas crianças no grupo I e $70 \%$ no grupo II. Foram observadas freqüentes anormalidades morfológicas e nos genes da tireoperoxidase e do receptor do TSH. Em nosso trabalho verificamos que 31 e 62 crianças, selecionadas como HC suspeitos, são comparáveis aos grupos I e II desse autor, respectivamente. Quando era observado nas crianças italianas um valor aumentado do TSH sérico (> $5 \mu \mathrm{U} /$ $\mathrm{mL}$ ) em duas diferentes medidas, a terapia com a levotiroxina era iniciada mesmo com T4 livre considerado normal. Futuro trabalho de nosso grupo avaliará se devemos assumir conduta semelhante.

Nosso percentual de $7,75 \%$ de pendências revela a existência de problemas sociais que requerem medidas administrativas, o que é relatado na literatura $(57,65)$.

Verificamos que as concentrações do TSHneo de nossas crianças $(1,16 \mu \mathrm{U} / \mathrm{mL} \pm 0,98 \mu \mathrm{U} / \mathrm{mL}$, tabela 2) são menores que dados publicados com a mesma tecnologia $2,70 \mu \mathrm{U} / \mathrm{mL} \pm 1,80 \mu \mathrm{U} / \mathrm{mL}$ (6) e do fabricante do kit 3,08 $\mu \mathrm{U} / \mathrm{mL} \pm 2,04 \mu \mathrm{U} / \mathrm{mL}$ (24). Quanto à variabilidade das concentrações do TSHneo, nossos resultados são maiores $(84,6 \% \mathrm{cv})$ do que a literatura estudada $(69,6 \% \mathrm{cv}$ e $66,2 \%$, respectivamente) talvez em razão da nossa maior variedade étnica e variação maior da idade da criança na coleta da triagem. Já nossos dados de TSHneo nas crianças triadas com 2 a 6 dias de idade, 1,33 $\pm 1,08 \mu \mathrm{U} / \mathrm{mL}$, são similares aos de crianças italianas, $1,4 \mu \mathrm{U} / \mathrm{mL} \pm 0,8 \mu \mathrm{U} /$ $\mathrm{mL}(29)$.

Averiguamos que a triagem neonatal foi realizada nos diversos quadrimestres nas diversas fases da triagem em Sergipe mais tardiamente que os programas de triagem de outros países $(3-5,18,23,26,28,29,32-35,38,3$ $9,41,43,44,51,52,63,64,67-80)$ que utilizam padrões de limite de normalidade para o TSHneo para idade na coleta aproximadamente de 2 a 6 dias e tempo para realização do ensaio aproximadamente de 3 dias, como também de centros de triagem nacionais $(36,37,42$, 45,46,59-62). A idade de nossas crianças nas coletas variou nos quadrimestres estudados de $9,9 \pm 7,7$ a 11,0 $\pm 12,6$ dias. Devemos considerar em relação à concentração do TSHneo e TSH, T4, T4 livre séricos em lactentes a influência da prematuridade, gemelaridade, síndrome de Down, baixo peso e muito baixo peso ao nascer, tipos de parto, patologias neonatais, auto-imunidade, mutações do receptor do TSH, baixas concen- 
trações de TBG, uso de drogas e proporção de HC transitório. O conjunto destes fatores condiciona peculiaridades específicas a cada região, que podem refletir em diferentes níveis de corte (19,60,66,77-80).

Em Sergipe, um estado litorâneo do Nordeste do Brasil e com baixa altitude, a temperatura, o iodo ambiental e o iodo alimentar podem reduzir a concentração do TSH das crianças do PTN $(47,52,54,55,80)$.

Desse modo, os resultados da triagem devem ser utilizados para definir os pontos de corte para o HC, levando-se em conta a idade da criança na coleta e devem ser adaptados a cada serviço. Os recém-nascidos classificados como suspeitos para $\mathrm{HC}$ devem ser acompanhados de perto pelos responsáveis pelos programas de triagem para evitar as conseqüências tardias do HC não tratado.

\section{AGRADECIMENTOS}

À Secretaria de Estado da Saúde de Sergipe e ao Núcleo de Pós-Graduação em Medicina da Universidade Federal de Sergipe (UFS).

\section{REFERÊNCIAS}

1. Foucault M. O nascimento da clínica. Tradução Roberto Machado. 2. ed. Rio de Janeiro: Forense-Universitária; 1980.

2. Gardner I. Endocrine and genetic diseases of childhood. Philadelphia and London: WB Saunders Company; 1969.

3. LaFranchi S. Detecção neonatal de hipotiroidismo congênito: Uma história de sucesso? Arq Bras Endocrinol Metab. 1995;39:80-8.

4. American Academy of Pediatrics. Update of newborn screening and therapy for congenital hypothyroidism. Pediatrics. 2006;117(6):2290-303.

5. Fisher DA, Klein AH. Thyroid development and disorders of thyroid function in the newborn. $\mathrm{N}$ Engl $\mathrm{J}$ Med. 1981;304(12):702-12.

6. Naruse H, Fukushi M, Takasugi N, Kunita S, Maeda S, Hamanaka $H$, et al. Neonatal hypothyroid screening by enzymeimmunossay for TSH. In: Medeiros-Neto G, Maciel RMB, Halpem A. lodine deficiency disorders and congenital hypothyroidism. São Paulo: EPM; 1986. p. 167-75.

7. Ford DH, Cramer EB. Developing nervous system in relation to thyroid hormones. In: Grave GD. Thyroid hormones and brain development. New York: Congress; Raven Press; 1977. p. 1-18.

8. Morreale de Escobar G, Escobar del Rey F, Obregon MJ. The hypothyroid rat. In: Medeiros-Neto GA. lodine deficiency disorders and congenital hypothyroidism. São Paulo: EPM; 1986.

9. Alberts B, Dennis B, Lewis J. Molecular biology of the cell. 3 . ed. New York: Garland-Puglisshing, Inc.; 1994.
10. Moschini L, Costa P, D'Addio A. II follow-up laboratoristico strumentale e clinico dell'ipotiroidismo congenito. Ann Ist Super Sanità. 1994;30(3):335-8.

11. Oppenheimer JH, Schwartz HL. Molecular basis of thyroid hormone-dependent brain development. Endocr Rev. 1997;18(4):462-75.

12. Weber G, Siragusa V, Rondanini GF, Cerai LMP, Mora S, Colombini J, et al. Neurophysiologic studies and cognitive function in congenital hypothyroid children. Pediatr Res. 1995;37(6):736-40.

13. Bellman SC, Davies A, Fuggle PG, Grant DB, Smith I. Mild impairment of neuro-otological function in early treated congenital hypothyroidism. Arch Dis Child. 1996;74:215-8.

14. Glorieux J, Desjardins M, Letarte J, Morissette J, Dussault JH. Useful parameters to predict the eventual mental outcome of hypothyroid children. Pediatr Res. 1988;24(1):6-8.

15. Tato L, Antoniazzi F, Costantini E, Zamboni G, Bertoldi C. La terapia dell ipotiroidismo congenito. Ann Ist Super Sanità. 1994;30(3):325-8.

16. Grant DB. Congenital hypothyroidism: optimal management in the light of 15 years experience of screening. Arch Dis Child. 1995;72:85-9.

17. Ehrlich RM. Thyroxin dose for congenital hypothyroidism. Clin Pediatr (Phila). 1995;34(10):521-2.

18. Dubuis JM, Glorieux J, Richer F, Deal CL, Dussault JH, Vliet GV. Outcome of severe congenital hypothyroidism: closing the developmental gap with early high dose levothyroxine treatment. J Clin Endocrinol Metab. 1996;81:222-7.

19. Bona G, Gallina MR, Zaffaroni M. Alterazioni transitorie della funzionalità tiroidea in período neonatale. Ann Ist Super Sanità. 1994;30(3):329-33.

20. Bargagna S. Prevenzione dei disturbi da ipotiroidismo congenito: un' esperienza di follow-up neuropsichico. Ann Ist Super Sanità. 1994;30(3):343-5.

21. Bottler J. Repercussões neurológicas do hipotireoidismo congênito. Arq Bras Endocrinol Metab. 1996;40(4):264-70.

22. Rodrigues PC. Bioestatística. 2. ed. Niterói: EDUFF; 1993.

23. Mendoza-Morfin F, Bucio-Delgado I, Bravo-Ríos LE, MonteroGonzález P, Gutiérrez-Avila C, Cárdenas-Tirado H, et al. Valores de referencia de triyodotironina, Tiroxina y tirotropina en niños prematuros sanos. Bol Med Hosp Infant Mex. 2001;58(1):6-11.

24. PerkinEImer Life Sciences, Wallac Oy, Turku, Finland. B032-312 AutoDelfia Neonatal hTSH, instructions for use: reagents for 1152 assays. 2002;12-4.

25. Pharoah POD, Madden MP. Audit of screening for hypothyroidism. Arch Dis Child. 1992;67:1073-6.

26. American Academy of Pediatrics. Newborn screening for congenital hypothyroidism: recommended guidelines. Pediatrics. 1993;91:1203-9.

27. Vasilevskaia IA, Guzeev GG, Baikov AD, Rykina IA, Bugrova VB. Clinical aspects of screening and diagnosis of congenital hypothyroidism in neonates in Moscow. Probl Endokrinol (Mosk). 1993;39(4):25-7.

28. Daliva A, Linder B, Martino-Nardij, Saenger P. Long term follow-up of borderline congenital hypothyroidism. Pediatric Academic Societies Annual Meeting. 1998;409.

29. Calaciura F, Motta RM, Miscio G, Fishera G, Leonardi D, Carta A, et al. Sub clinical hypothyroidism in early childhood: a frequent outcome of transient neonatal hyperthyrotropinemia. $J$ Clin Endocrinol Metab. 2002;87(7):3209-14 
30. Lott JA, Sardovia-lyer M, Speakman KS, Lee KK. Age-dependent cutoff values in screening newborns for hypothyroidism. Clin Biochem. 2004;37(9):791-7.

31. Suolinna EM, Torresani TE, Westgard JO. Designing quality control for neonatal screening assays. Southeast Asian J Trop Med Public Health. 2003;34 Suppl 3:103-6.

32. Tyfield IA, Abusrewil SSA, Jones SR, Savage DLC. Persistent hyperthyrotropinaemia since the neonatal period in clinically euthyroid children. Eur J Pediatr. 1991;150:308-9.

33. Sorcini M, Fazzini C, Oliveiri A, Grandolfo ME, Medda E, Stazi $\mathrm{MA}$, et al. Lo screening neonatale dell ipotiroidismo congenito. II registro nazionale. Ann Ist Super Sanità. 1994;30:275-87.

34. Saslow JG, Post EM, Southard CA. Thyroid screening for early discharged infants. Pediatrics. 1996;98:41-3.

35. Oyarzábal M, Chueca M, Elso J, Sola A. Screening neonatal del Hipotiroidismo congénito: resultados del programa en $\mathrm{Na}$ varra. An Sist Sanit Navar. 1998;21:6-15.

36. Nascimento ML, Pires MMS, Nassar SM, Ruthland L. Avaliação do programa de rastreamento neonatal para o hipotireoidismo congênito da Secretaria de Estado da Saúde de Santa Catarina. Arq Bras Endocrinol Metab. 2003;47(1):75-81.

37. Ramalho RJR, Ramalho ARO, Oliveira CRP, Aguiar-Oliveira $\mathrm{MH}$. Evolução do programa de triagem neonatal para o hipotireoidismo congênito e fenilcetonúria no Estado de Sergipe de 1995 a 2003. Arq Bras Endocrinol Metab. 2004;48(6):890-6.

38. Alm J, Hagenfeldt L, Larsson A, Lundberg K. Incidence of congenital hypothyroidism: retrospective study of neonatal laboratory screening versus clinical symptoms as indicators leading to diagnosis. Br Med J. 1984;2893:1171-4.

39. Walfish PG. Thyroid function in pediatrics. In: Hicks JM, Boeckx RL, editores. Pediatric clinical chemistry. Philadelphia; 1984.

40. Weiner R, Utiger RD, Lew R, Emerson $\mathrm{CH}$. Age, sex and serum thyrotropin concentrations in primary hypothyroidism. Acta Endocrinol (Copenh). 1991;124(4):364-9.

41. Fisher DA. Clinical review 19. Management of congenital hypothyroidism. J Clin Endocrinol Metab. 1991;72:523-9.

42. Jardim LB, Leite JCL, Silveira EL, Barth ML, Giugliani R. Resultados preliminares de um programa de detecção precoce para o hipotireoidismo congênito no Rio Grande do Sul. J Pediatr (Rio J).1992;68(7/8):239-42.

43. Majeed-Saidan MA, Joyce B, Khan M, Hamam HD. Congenital hypothyroidism: the riyadh military hospital experience. Clin Endocrinol (Oxf). 1993;38:191-5.

44. Kreisner E, Schermann L, Camargo-Neto E, Gross JL. Predictors of intellectual outcome in a cohort of brazilian children with congenital hypothyroidism. Clin Endocrinol (Oxf). 2004;60:250-5.

45. Ramos AJS, Rocha AM, Costa ADM, Benício AVL, Ramos ALC, Silva CRA, et al. Avaliação do programa de rastreamento de doenças congênitas em Campina Grande-PB, Brasil. Arq Bras Endocrinol Metab. 2003;47(3):280-4.

46. Almeida AM, Godinho TM, Teles MS, Rehem AP, Jalil HM, Fukuda TG, et al. Avaliação do programa de triagem neonatal na Bahia no ano de 2003. Rev Bras Saúde Matern Infant. 2006;6(1):85-91.

47. Veiga CMR, Monteiro CBA, Fonseca AA, Carvajal S, Guimarães MM. Variação sazonal nos níveis de TSH em testes de rastreamento para o hipotireoidismo congênito. J Pediatr (Rio J). 1988;74(5):383-8.

48. Grandolfo ME, Sagliocca L, Stazi MA, Medda E, Oliviere A, Sorcini M. Valutazione dei fattori di rischio dell'ipotiroidismo congenito: prospective di uno studio caso-controllo. Ann Ist Super Sanità. 1994;30(3):295-8.

49. Toublanc JE. Comparison of epidemiological data on congenital hypothyroidism in Europe with those of other parts in the world. Horm Res. 1992;38:230-5.

50. Chiovato L, Lapi P, Santini F, Bona G, Weber G, Siragusa V, et al. Thyroid autoimmunity and congenital hypothyroidism. Ann Ist Super Sanità. 1994;30(3):317-23.

51. Dunn JT, Haar FVD. A practical guide to the correction of iodine deficiency. Netherlands: International Council for Control of lodine Deficiency Disorders. Unicef; 1990.

52. Oliveri A, Fazzini C, Grandolfo ME, Stazi MA, D'Achivio M, De Angelis $\mathrm{S}$, et al. Ipotiroidismo congênito transitório in aree iodocarenti. Ann Ist Super Sanità. 1994;34:331-6.

53. Duarte GC, Tomimori EK, Boriolli RA, Ferreira JE, Catarino RM, Camargo GMN. Avaliação ultra-sonográfica da tireóide e determinação da iodúria em escolares de diferentes regiões do estado de São Paulo. Arq Bras Endocrinol Metab. 2004;48(6):842-8.

54. Alves ML, Maciel RMB, Kunii I, lazigi N. Correlação entre níveis de iodúria e TSH colhido em cordão umbilical de recémnascidos do Hospital das Clínicas da Faculdade de Medicina de Ribeirão Preto, São Paulo. Arq Bras Endocrinol Metab. 2005;49(4):516-20.

55. Doménech Martínez E, Barroso Guerrero F. Utilidad del programa de cribado neonatal del hipotiroidismo congénito para la valoración de la deficiencia de yodo en Canarias. An Pediatr. 2003;58(4):357-63.

56. Copeland DL, Sullivan KM, Houston R, May W, Mendoza I, Salamatullah $\mathrm{Q}$, et al. Comparison of neonatal thyroid-stimulating hormone levels and indicators of iodine deficiency in school children. Public Health Nutrition. 2002:5(1);81-7.

57. Fisher D. Eficácia dos programas de rastreamento neonatal para hipotireoidismo congênito: prevalência de casos não identificados. São Paulo: Interlivros; 1987.

58. Yunis KA, Nasr MR, Lepejian G, Najjar S, Daher R. False-negative primary neonatal thyroid screening: the need for clinical vigilance and seconary screening. J Med Screen. 2003;10:2-4.

59. Ramalho RJR, Valido DP, Oliveira MH. Avaliação do programa de triagem para o hipotireoidismo congênito no estado de Sergipe. Arq Bras Endocrinol Metab. 2000;44(2):157-61.

60. Silva LO, Dias VMA, Silva IN, Chagas AJ. Hipotiroidismo congênito transitório: perfil das crianças identificadas no programa estadual de triagem neonatal de Minas Gerais, Brasil. Arq Bras Endocrinol Metab. 2005;49(4):61-70.

61. Nesi-França S. Triagem neonatal para hipotireoidismo congênito no Estado do Paraná. In: Medeiros-Neto, G, editor. Hipotireoidismo congênito no Brasil: como era, como estamos, para onde vamos. São Paulo: Laramara; 2004. p. 47-9.

62. Nascimento M. Hipotireoidismo congênito em Santa Catarina. In: MEDEIROS-NETO G, editor. Hipotireoidismo congênito no Brasil: como era, como estamos, para onde vamos. São Paulo: Laramara; 2004. p. 53-61.

63. Marrero-González N, Rodrigues-Fernandez C, Hipotiroidismo congênito: historia e impacto del tamizaje. Rev Biomed. 2000;11:283-92.

64. Abodovsky NG, Vivanco XW, Cuello XA, Pérez PO, Lobo GS, Brantes JM, et al. Detección precoz de hipotiroidismo congênito en Chile. Resultados en 24 meses. Rev Chil Pediatr. 1995;66(3):140-4.

65. Wu XY, Wang H, Huang DM, Lei HX, Hu R, Ma L, et al. Screening for congenital hypothyroidism in 106.224 neonates in hunan province. Beijing Da Xue Xue Bao. 2005;37(1):42-4. 
66. Fisher DA. Editorial: next generation newborn screening for congenital hypothyroidism. J Clin Endocrinol Metab. 2005;90 (6):3797-9.

67. Fisher DA. Congenital hypothyroidism. Thyroid International 2002; 3:2-10 [acesso em 2008 Jan 2]. Disponível em: http:// abonnieren.merck.de/servlet/PB/menu/1271570/index.html.

68. González VG, Santucci ZC, Pattin J, Apezteguía M, Borrajo G. Programa de pesquisa neonatal de hipotiroidismo congénito de la provincia de Buenos Aires: 1.377 .455 niños evaluados en diez años de experiencia. Arch Argent Pediatr 2007;105(5):390-7.

69. Massoud A, Mahin H, Ramin I, Silva H, Sassan H, Khosro. Rate of recalls in congenital hypothyroidism based upon a regiona survey in Isfahan, Iran, using serum t4 and tsh analyses: comparison of two different recall methods. Horm Res. 2005;64: 287-92.

70. Wilshaw ME, Villarroel CF, Parodi BQG, Lamas RC, Valdés TMHG. Detección precoz de hipotiroidismo congénito. Rev Chil Pediatr. 1986;57(6):528-30.

71. LaFranchi $\mathrm{SH}$, Austin J. How should we be treating children with congenital hypothyroidism? J Pediatr Endocrinol Metab. 2007;20(5):559-78.

72. Jones JH, Mackenzie J, Craft GA, Beaton S, Young D, Donaldson MD. Improvement in screening performance and diagnosis of congenital hypothyroidism in Scotland 1979-2003. Arch Dis Child. 2006;91(8):680-5.

73. Willi SM, Moshang T Jr. Diagnostic dilemmas: results of screening tests for congenital hypothyroidism. Pediatr Clin North Am. 1991;38(3):555-66.

74. Marrero-Gonzalez N, Gonzalez-Reyes EC, Frómeta-Suarez A, Baloy-Nodarse A, Castells-Martinez E, Pérez-Morás PL, et al. Influencia de la edad em los resultados del cribado neonata de hipotiroidismo congénito, fenilcetonuria y galactosemia. Rev Mex Patol Clin. 2004;51(4):220-5.
75. Cuello AX, Pérez OP, Vivanco WX, Lobos SG, Bruggendiek MB, Pérez R A. Evaluación de 7 años de programa nacional de detección precoz de hipotiroidismo congénito en Chile. Bol Hosp San Juan de Dios. 2004;51(5):221-30.

76. Costante G, Grasso L, Ludovico O, Marasco MF, Nocera M, Schifino $E$, et al. The statistical analysis of neonatal TSH results from congenital hypothyroidism screening programs provides a useful tool for the characterization of moderate iodine deficiency regions. J Endocrinol Invest. 1997;20(5):251-6.

77. Kaye Cl. Newborn screening fact sheets. Pediatrics. 2006;118 (3):934-63.

78. Trotsenburg ASP, Vulsma HMT, Santen WC, Vijlder JJM. Lower neonatal screening thyroxine concentrations in down syndrome newborns. J Clin Endocrinol Metab. 2003,88(4):1512-5.

79. Kempers $\mathrm{MJE}$, Lanting $\mathrm{Cl}$, van Heijst $\mathrm{AFJ}$, van Trotsenburg ASP, Wiedijk BM, Vijlder JJM, et al. Neonatal screening for congenital hypothyroidism based on thyroxine, thyrotropin and thyroxine-binding globulin measurement: potentials and pitfalls. J Clin Endocrinol Metab. 2006;91(9):3370-6.

80. Radzivil TT, Krat IV. Neonatal monitoring of congenital hypothyroidism in a mild iodine-deficiency region. Klin Lab Diagn. 2006;(7):9-11.

\section{Endereço para correspondência:}

Manuel H. Aguiar-Oliveira

Departamento de Medicina do Hospital Universitário

Rua Cláudio Batista s/n

49060-100 Sanatório Aracaju, SE

E-mail: herminio@infonet.com.br 